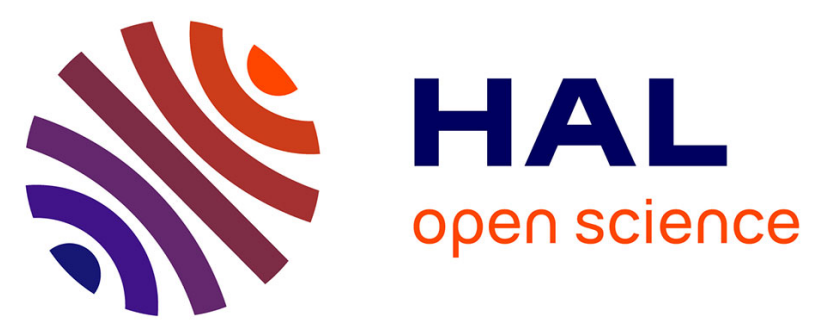

\title{
Orientation-Dependent Proton Relaxation of Water Molecules Trapped in Solids: Crystallites with Long-Lived Magnetization
}

Diego Carnevale, Sina Marhabaie, Philippe Pelupessy, Geoffrey Bodenhausen

\section{- To cite this version:}

Diego Carnevale, Sina Marhabaie, Philippe Pelupessy, Geoffrey Bodenhausen. Orientation-Dependent Proton Relaxation of Water Molecules Trapped in Solids: Crystallites with Long-Lived Magnetization. Journal of Physical Chemistry A, 2019, 123 (45), pp.9763-9769. 10.1021/acs.jpca.9b07303 . hal02407818

\author{
HAL Id: hal-02407818 \\ https://hal.sorbonne-universite.fr/hal-02407818
}

Submitted on 12 Dec 2019

HAL is a multi-disciplinary open access archive for the deposit and dissemination of scientific research documents, whether they are published or not. The documents may come from teaching and research institutions in France or abroad, or from public or private research centers.
L'archive ouverte pluridisciplinaire HAL, est destinée au dépôt et à la diffusion de documents scientifiques de niveau recherche, publiés ou non, émanant des établissements d'enseignement et de recherche français ou étrangers, des laboratoires publics ou privés. 


\title{
Orientation-Dependent Proton Relaxation of Water Molecules Trapped in Solids: Crystallites with Long-Lived Magnetization
}

\author{
Diego Carnevale, ${ }^{*}$ Sina Marhabaie, Philippe Pelupessy and Geoffrey Bodenhausen \\ Laboratoire des biomolécules, LBM, Département de chimie, École normale supérieure, PSL University, Sorbonne \\ Université, CNRS, 75005 Paris, France
}

\section{Corresponding author: Diego Carnevale \\ diego.carnevale@ens.fr}

Accepted on J Phys Chem A

DOI: 10.1021/acs.jpca.9b07303 


\begin{abstract}
The longitudinal spin-lattice relaxation properties of water molecules trapped in a static powdered polycrystalline sample of barium chlorate monohydrate are investigated by means of solid-state ${ }^{1} \mathrm{H}$ NMR spectroscopy. Different portions of the inhomogeneous Pake pattern that are associated with crystallites at different orientations with respect to the external magnetic field show either a mono- or a bi-exponential recovery. At high field (9.4 T), the chemical shift anisotropy is the main interaction that is responsible for the inhomogeneity of the relaxation rates. A theoretical description of rapid two-site hopping about the $\mathrm{H}-\mathrm{O}-\mathrm{H}$ bisector in the framework of Liouville space agrees very well with the experimental evidence. Numerical simulations predict a distribution of mono-exponential time constants associated with individual single-crystal orientations. Overlapping signals give rise to bi-exponential recovery. This is confirmed experimentally by ${ }^{1} \mathrm{H}$ NMR spectra of static single crystals.
\end{abstract}




\section{Introduction}

Water is not only essential to all living organisms, but also found in mineral phases such as wadsleyite and ringwoodite that are constituents of the Earth's lower mantle. ${ }^{1-3}$ The knowledge of the amount of water trapped in the Earth's interior is important to understand and analyze the propagation of seismic waves. ${ }^{4,5}$ The study of hydrates is crucial to understand polymorphism in pharmaceutical formulations. ${ }^{6,7}$ These affect drug solubility and stability and are therefore of vital importance for pharmaceutical industry. Water is also at the very core of technologies such as MRI, where local variations of relaxation rates of $\mathrm{H}_{2} \mathrm{O}$ molecules are responsible for contrast in images used for diagnostic purposes. ${ }^{8}$ Despite its ubiquity and essential functions, some properties of water still remain unclear. Dynamic processes such as hopping about the two-fold axis of water molecules, tunneling, ${ }^{9-11}$ and chemical exchange between neighboring water molecules make studies of some of the most basic properties of $\mathrm{H}_{2} \mathrm{O}$ molecule remarkably challenging.

Longitudinal relaxation of nuclear magnetization in solid samples is known to be orientation dependent. ${ }^{12}$ Understanding the orientation dependence of the relaxation time constants $T_{1}$ is crucial for the identification of para water in solid materials, both at room temperature and at spin temperatures in the $\mathrm{mK}$ range that can be achieved by dynamic nuclear polarization, where the NMR-invisible singlet state can be depleted with respect to the observable triplet state. ${ }^{13}$ As the overpopulation of the singlet state by means of $r f$-pulse schemes requires non-equivalent spin pairs, the chemical shift anisotropy (CSA) ${ }^{14}$ offers a means to lift the degeneracy between the two otherwise equivalent protons of a water molecule. This may provide access to the singlet-triplet manifolds ${ }^{15-17}$ in view of creating imbalances. Furthermore, dynamics in solid samples may result in modulations of the CSA interactions that affect the relaxation properties of water molecules trapped in solids.

We report a proton NMR relaxation study of water molecules in a static powdered sample of barium chlorate monohydrate $\left(\mathrm{Ba}\left(\mathrm{ClO}_{3}\right)_{2} \cdot \mathrm{H}_{2} \mathrm{O}\right)$ in a field $B_{0}=9.4 \mathrm{~T}$ at room temperature $T=298 \mathrm{~K}$. This system offers a unique opportunity to study water relaxation properties as $\mathrm{H}_{2} \mathrm{O}$ molecules are located at a sufficient distance from one another in the unit cell 
so as to result in a quasi-ideal Pake pattern ${ }^{18}$ in static solid-state NMR spectra at low fields. In addition to the predominant intramolecular ${ }^{1} \mathrm{H}-{ }^{1} \mathrm{H}$ dipolar couplings of $c a .30 \mathrm{kHz}$, smaller interactions such as the CSA (ca. 15 ppm or $6 \mathrm{kHz}$ at $9.4 \mathrm{~T}$ ) that affect the Pake pattern can be investigated by means of NMR spectroscopy as they result in a clear asymmetry of the 'shoulders' of the powder pattern at high fields. ${ }^{19-22}$ Furthermore, peculiar cross-term splittings which stem from the orientational inequivalence of the two proton CSA tensors have been recently observed by ${ }^{1} \mathrm{H}$ MAS NMR spectroscopy at temperatures below $150 \mathrm{~K} .{ }^{23}$

In this study, the orientation dependence of $T_{1}$ across the static lineshape is determined experimentally and rationalized theoretically. In some parts of the Pake pattern, the relaxation behavior is bi-exponential, in other parts it is mono-exponential. The main interaction that is responsible for the orientation-dependence of $T_{1}$ is found to be the CSA. The distribution of $T_{1}$ time constants across the Pake pattern is interpreted by a theoretical treatment in Liouville space to describe rapid two-fold hopping about the $\mathrm{H}-\mathrm{O}-\mathrm{H}$ bisector that averages the CSA tensors. The wide spread of $T_{1}$ values observed near the 'horns' of the Pake pattern is shown to obey simple bi-exponential laws, whereas the narrower distribution of $T_{1}$ values near the 'shoulders' and in the center of the Pake pattern obeys a mono-exponential behavior. Weak long-range intermolecular couplings and minor librations give rise to a small isotropic contribution to the predominantly orientation-dependent relaxation rates. We show that at some orientations, the magnetization of ortho water can have life-times $T_{1}$ that are comparable to the time constant $T_{S}$ for para-to-ortho interconversion observed for water in fullerene cages at room temperature.

\section{Experimental Section}

All spectra were recorded on a wide-bore Bruker 400 spectrometer (9.4 T) with an AvanceIII console, using non-spinning $4 \mathrm{~mm} \mathrm{ZrO}_{2}$ rotors in a double-resonance MAS probe. The rf-field amplitude for proton pulses was $v_{1}=100 \mathrm{kHz}$, corresponding to a $90^{\circ}$ pulse length of $\tau_{p}=2.5 \mu \mathrm{s}$. A recovery delay of $30 \mathrm{~s}$ was used in all cases, although this is not sufficient for $T_{1}{ }^{(\max )} \approx 40 \mathrm{~s}$. The final $90^{\circ}$ pulse of inversion recovery experiments was replaced by a $90^{\circ}-\tau$ - $90^{\circ}$ - $\tau$ - solid echo with $p=0 \rightarrow p=+1 \rightarrow p=-1$ coherence pathway selection using a four-step phase cycle ${ }^{24}$ so as to i) 
eliminate strong background signals and ii) refocus both shielding and dipolar interactions that would otherwise result in distorted lineshapes. ${ }^{25}$ The sample of $\mathrm{Ba}\left(\mathrm{ClO}_{3}\right)_{2} \cdot \mathrm{H}_{2} \mathrm{O}$ was purchased from Sigma-Aldrich and used without purification. The powder was kept in an oven overnight at $80^{\circ} \mathrm{C}$ to remove loosely bound water that would otherwise result in a sharp isotropic peak in the center of the Pake pattern. Single crystals were grown by allowing a saturated aqueous solution of barium chlorate monohydrate to dry out over a few days at room temperature. For the spectra of Fig. 5, a single crystal was roughly cut to a cube so as to be able to move freely in a 4 $\mathrm{mm}$ rotor. Ejection and insertion cycles of this rotor were used to generate 'random' orientations of the crystal with respect to the static field.

\section{Results and discussion}

Figure 1(a) shows experimental ${ }^{1} \mathrm{H}$ NMR spectra obtained by inversion-recovery experiments combined with dipolar echoes. The initial and final lineshapes correspond to almost perfectly inverted and nearly fully relaxed magnetization. The variation of the lineshapes as a function of the recovery delay indicates a dramatic orientation dependence of $T_{1}$ relaxation time constants across the Pake pattern. A spectrum acquired near the zero-crossing at $\tau_{\text {rel }}=10 \mathrm{~s}$ is highlighted in red. This spectrum clearly shows that some portions of the lineshape (positive signals near the 'shoulders' and 'horns') recover faster than others (negative signals between the 'horns'). Moreover, the central part of the Pake pattern, associated with crystallites oriented so that the $\mathrm{H}-\mathrm{H}$ vector lies near to the magic angle $\left(\beta=54.74^{\circ}\right)$ with respect to the static field, relaxes faster than neighboring orientations. In Figure 1(b), black data points indicate the integrals of the spectra of (a) as a function of $0<\tau_{\text {rel }}<350 \mathrm{~s}$. When attempting to fit this recovery to a mono-exponential function, the curve shown by the dashed black line was obtained $\left(T_{1}{ }^{\text {(app })}=16.3 \pm 0.5 \mathrm{~s}\right)$. Clearly, such a model is not compatible with the experimental evidence. When fitting to a bi-exponential function of the form $I\left(\tau_{\text {rel }}\right)=A_{0}-A_{1} \exp \left\{-\tau_{\text {rel }} / T_{1}{ }^{(1)}\right\}-$ $A_{2} \exp \left\{-\tau_{\text {rel }} / T_{1}{ }^{(2)}\right\}$, a much better agreement is obtained, as shown by the continuous red line $\left(T_{1}{ }^{(1)}=10.3 \pm 0.3 \mathrm{~s}\right.$ and $T_{1}^{(2)}=33.5 \pm 1.5 \mathrm{~s}$, with relative weights $A_{1}=0.62 \pm 0.03$ and $A_{2}=0.38 \pm$ 0.03). A semi-logarithmic plot of the same data points of Fig. 1(b) is shown in the inset. It is worth mentioning that $\mathrm{x}$-ray and neutron diffraction reveal only a single crystallographically 
distinct water molecule in the unit cell. ${ }^{26,27}$ This latter fact is also in agreement with data reported in the literature about solid-state NMR studies of ${ }^{2} \mathrm{H},{ }^{1} \mathrm{H}$ and ${ }^{17} \mathrm{O}$ in barium chlorate monohydrate. ${ }^{28-31}$ In order to interpret the experimental evidence of a bi-exponential relaxation for water magnetization, we consider the theoretical framework introduced by Torchia and Szabo ${ }^{12}$ and Vold and Vold ${ }^{32}$ that describe the orientation dependence of $T_{1}$ in solid-state NMR. The main orientation-dependent interaction in our system is the intramolecular dipolar coupling between the two protons in each water molecule. These protons are ca. $1.6 \AA$ apart, resulting in a dipolar coupling constant $b_{i j} \approx 29 \mathrm{kHz}$. Relaxation requires that spin transitions be induced by fluctuating interactions. Twofold flips about the $\mathrm{H}-\mathrm{O}-\mathrm{H}$ bisector cannot contribute to relaxation, since the dipolar interaction is invariant to $180^{\circ}$ rotations. Therefore, other interactions need to be invoked to explain the experimental evidence. We have characterized the proton CSA interaction in the same system by means of both static and MAS techniques. ${ }^{22}$ The experimental findings in our previous studies were in excellent agreement with DFT calculations based on planewave-pseudopotential methods and indicate a CSA tensor with $\Delta_{c s}=\delta_{z z}-\delta_{\text {iso }}=-15.4 \mathrm{ppm}$ and $\eta_{c s}=\left(\delta_{y y}-\delta_{x x}\right) / \Delta_{c s}=0.1$ in the absence of motions. At room temperature, the CSA tensors are partly averaged by rapid $180^{\circ}$ rotations about the $\mathrm{H}-\mathrm{O}-\mathrm{H}$ bisector, leading to average values $\bar{\Delta}_{C S}=-7.6 \mathrm{ppm}$ and $\bar{\eta}_{C S}=1.0$. As the intermolecular dipolar couplings with neighboring water molecules do not exceed $c a .1 .6 \mathrm{kHz}$ (more than an order of magnitude smaller than the intramolecular couplings), we conclude that the CSA must be the dominant relaxation mechanism that is responsible for the orientation dependence of relaxation shown in Fig. 1(a-b). Long-range intermolecular dipolar couplings and small librations about the $\mathrm{H}-\mathrm{O}-\mathrm{H}$ bisector give rise to small contributions to the relaxation rates that we shall assume to be isotropic.

According to this model, the relaxation time constants $T_{1}{ }^{(1)}$ and $T_{1}{ }^{(2)}$ must depend on two Euler angles that express the orientation of the averaged CSA tensors with respect to the external magnetic field. Obviously, as stated by Torchia, this implies that "it is not possible to ascribe a single $T_{1}$ value at a particular position within inhomogeneous lineshapes". The relevant expression for the relaxation rates is: ${ }^{33}$ 
$R_{1}=\frac{1}{T_{1}}=\frac{\left(\Delta_{C S} \omega_{0}\right)^{2}}{2} J_{1}\left(\omega_{0}\right)$

assuming for simplicity that the rhombicity of the CSA tensors can be neglected $\left(\eta_{C S}=0\right)$ and expressing $\Delta_{c s}$ in ppm. For hopping about the $\mathrm{H}-\mathrm{O}-\mathrm{H}$ bisector through $180^{\circ}$, the orientation dependence of the spectral density $J_{1}\left(\omega_{0}\right)$ is given by: $:^{32}$

$J_{1}\left(\omega_{0}\right)=\frac{1}{2} \frac{3 k_{e x}}{4 k_{e x}^{2}+\omega_{0}^{2}} \sin ^{2} \beta \cos ^{2} \beta\left[1-3 \cos ^{2} \beta_{C R}+4 \cos ^{4} \beta_{C R}+\left(4 \cos ^{4} \beta_{C R}-5 \cos ^{2} \beta_{C R}+1\right) \cos 2 \alpha_{C R}\right]$,

where $k_{e x}$ is the exchange rate for the two-fold flip. The angle $\beta$ describes the relative orientations of the main axes of the non-averaged CSA tensors with respect to the $\mathrm{H}-\mathrm{O}-\mathrm{H}$ bisector and amounts to $54.3^{\circ}$ in our case, i.e., very close to the magic angle. ${ }^{29}$ The angles $\beta_{C R}$ and $\alpha_{C R}$ relate the crystal frame $C$ to the rotor frame $R$ and must be averaged over all orientations in the powder. The usual subsequent transformation to the lab frame $L$ is irrelevant in static experiments. A more general expression, which includes the effects of the asymmetry $\eta_{c s}$ on $J_{1}\left(\omega_{0}\right)$, may be found in the literature ${ }^{32}$ but for simplicity will not be used in this study.

Figure 2(a) shows a contour plot of the function $J_{1}\left(\omega_{0}\right)$ of Eq. 2 versus $\beta_{C R}$ and $\alpha_{C R}$, assuming a fast exchange rate of two-fold hopping $k_{e x}=4.5 \times 10^{9} \mathrm{~s}^{-1}$ at $298 \mathrm{~K}^{23}$ and $\omega_{0}=400$ $\mathrm{MHz}$. Figure 2(b) shows a three-dimensional plot of the same function. Two regions in the ( $\beta_{C R}$, $\left.\alpha_{C R}\right)$ space, in particular, near $\left(45^{\circ}, 0^{\circ}\right)$ and $\left(90^{\circ}, 90^{\circ}\right)$, show remarkably long $T_{1}$ values beyond 40 s. In contrast, subspaces where the magnetization is short lived, with $T_{1}$ values below $10 \mathrm{~s}$, can be found near $\left(90^{\circ}, 0^{\circ}\right)$ and in a vertical band $\left(\left[0^{\circ}-30^{\circ}\right],\left[0^{\circ}-90^{\circ}\right]\right)$. A narrow saddle point or 'sweet spot' with $T_{1} \approx 25 \mathrm{~s}$ is found in the region $\left(62^{\circ},\left[0^{\circ}-90^{\circ}\right]\right)$. Importantly, the contour plot shows that, for different values of $\beta_{C R}$, i.e., for different positions within the Pake spectrum, the $T_{1}$ values vary in a range from 10 to $40 \mathrm{~s}$.

In order to further investigate the consequences of these predictions, vertical cross sections were taken from Fig. 1(a) and analyzed to extract the relevant relaxation parameters across the Pake pattern. The corresponding data is shown in Figure 3(a). Interestingly, a biexponential function is clearly required to model the inversion-recovery data near the 'horns' of 
the Pake pattern. In contrast, mono-exponential functions (green dots) suffice near the shoulders and near the center of the Pake pattern, where the latter correspond to crystals oriented near the magic angle $\beta_{C R}=\arctan \left(2^{1 / 2}\right)=54.74^{\circ}$. This finding can be rationalized by considering how doublets associated with individual crystallites contribute to the Pake pattern: (i) between the horns, pairs of different crystallite orientations with different relaxation time constants result in identical splittings due to the homonuclear dipole-dipole coupling; (ii) this does not give rise to significantly different relaxation time constants around the magic angle; and (iii) only dipolar doublets with a single relaxation time constant contribute to the shoulders outside the horns.

Figure $3(b)$ shows how the weights $A_{1}$ and $A_{2}$ of the bi-exponential components obtained by fitting the data of Fig. 1(a), are scattered across the Pake pattern. The sum $A_{1}+A_{2}$ of these weights, indicated by black points, closely resembles the Pake pattern and testifies for the overall quality of the individual fits. It is interesting to note that, near the 'horns' of the Pake pattern, $T_{1}{ }^{(2)} \approx 40 \mathrm{~s}$ is comparable if not longer than the singlet-state time constant $T_{\mathrm{S}}$ reported for para-to-ortho conversion of water confined in fullerene $\mathrm{C}_{60}$ cages. ${ }^{34}$ In our case, each water molecule undergoes continuous disruption and reestablishment of two $\mathrm{H}$-bonds with chlorate oxygen atoms as a result of rapid hopping about the $\mathrm{H}-\mathrm{O}-\mathrm{H}$ bisector at room temperature. The resulting fluctuations of the CSA interaction near the Larmor frequency lead to the observed relaxation. In contrast, the dominant relaxation mechanism for freely tumbling water molecules confined in fullerene cages is spin rotation, since the water molecules are only subject to vibrational motions with frequencies on the order of $10^{13} \mathrm{~s}^{-1}>\omega_{0}$ that are not expected to make a significant contribution to longitudinal relaxation. ${ }^{29,34}$

The presence of the CSA interaction renders the assignment of specific dipolar doublet splittings in the Pake pattern to particular sets of Euler angles more complicated than for the simple case where only dipolar interactions are present. In the latter case, varying a single angle $\beta_{C R}$ suffices to accurately reconstruct the full Pake pattern by associating any position within the pattern to one or at most two $\beta_{C R}$ values. In contrast, in the case at hand, the presence of the CSA interaction requires a second angle $\alpha_{C R}$ to fully describe the powder average. In order to 
take this situation into account, we analyzed our experimentally observed profiles in a Liouville space framework where both rapid chemical exchange due to fast hopping and inhomogeneous longitudinal relaxation rates due to the CSA are taken into account. The evolution of populations and coherences can be evaluated by means of the master equation: ${ }^{35,36}$

$\boldsymbol{\rho}(t)=\exp \left\{\left(-i L_{c o h}+L_{i n c o h}\right) \Delta t\right\} \boldsymbol{\rho}(0)$,

where $L_{c o h}$ is the commutation superoperator that describes coherent effects and $L_{\text {incoh }}$ takes into account incoherent effects, namely, chemical exchange and orientation-dependent relaxation. The recovery of Zeeman order can be fully described in the basis $B^{\prime}=\left\{I_{z}, S_{z}\right\}$ with the corresponding superoperator:

$L_{\text {incoh }}^{\prime}=\left(\begin{array}{cc}R_{1, I}+k_{e x} & -k_{e x} \\ -k_{e x} & R_{1, S}+k_{e x}\end{array}\right)$,

with $R_{1, l}=R_{1, S}=R_{1}$ of Eq. (1). The simulation of the subsequent solid echo requires a singlequantum subspace spanned by the basis $B^{\prime \prime}=\left\{I_{x}, I_{y}, S_{x}, S_{y}, 2 I_{x} S_{z}, 2 I_{y} S_{z}, 2 I_{z} S_{x}, 2 I_{z} S_{y}\right\}$ with the following superoperator:

$$
L_{\text {incoh }}^{\prime \prime}=\left(\begin{array}{cccccccc}
k_{e x} & 0 & -k_{e x} & 0 & 0 & 0 & 0 & 0 \\
0 & k_{e x} & 0 & -k_{e x} & 0 & 0 & 0 & 0 \\
-k_{e x} & 0 & k_{e x} & 0 & 0 & 0 & 0 & 0 \\
0 & -k_{e x} & 0 & k_{e x} & 0 & 0 & 0 & 0 \\
0 & 0 & 0 & 0 & k_{e x} & 0 & -k_{e x} & 0 \\
0 & 0 & 0 & 0 & 0 & k_{e x} & 0 & -k_{e x} \\
0 & 0 & 0 & 0 & -k_{e x} & 0 & k_{e x} & 0 \\
0 & 0 & 0 & 0 & 0 & -k_{e x} & 0 & k_{e x}
\end{array}\right) \text {, }
$$

where transverse relaxation is neglected. Longitudinal recovery of Zeeman order can be evaluated within the $B^{\prime}$ subspace, starting from the initial state $\rho^{\prime}(0)=\{-1,-1\}$, to evolve according to Eq. (3) during the time interval $\tau_{\text {rel. }}$ Subsequently, transverse magnetization is allowed to evolve during the $90^{\circ}-\tau-90^{\circ}-\tau$ - solid echo sequence within the $B^{\prime \prime}$ subspace after a change of the basis $\rho^{\prime \prime}(0)=\left\{\rho^{\prime}\left(\tau_{\text {rel }}\right)_{1}, 0, \rho^{\prime}\left(\tau_{\text {rel }}\right)_{2}, 0,0,0,0,0\right\}$ that mimics the effect of the first 
(ideal) $90^{\circ}$ pulse. A further change of basis $\rho^{\prime \prime \prime}(\tau)=\left\{\rho^{\prime \prime}(\tau)_{1},-\rho^{\prime \prime}(\tau)_{2}, \rho^{\prime \prime}(\tau)_{3,}, \rho^{\prime \prime}(\tau)_{4,}, \rho^{\prime \prime}(\tau)_{5}\right.$, $\left.\rho^{\prime \prime}(\tau)_{6},-\rho^{\prime \prime}(\tau)_{7}, \rho^{\prime \prime}(\tau)_{8}\right\}$ was utilized to simulate the $p=1 \rightarrow p=-1$ coherence selection performed by the second (ideal) $90^{\circ}$ pulse. Finally, a further change to the basis $\rho^{\prime \prime \prime \prime}(0)=$ $\left\{\rho^{\prime \prime \prime}(2 \tau)_{1}, 0, \rho^{\prime \prime \prime}(2 \tau)_{3}, 0,0,0,0,0\right\}$ was performed at the end of the echo, prior to detection.

Figure $3(\mathrm{c})$ shows the relaxation time constants $T_{1}{ }^{(1)}$ and $T_{1}{ }^{(2)}$ calculated across the Pake pattern by simulating an inversion-recovery experiment combined with a dipolar echo sequence by means of Eq. (3). The quantitative agreement with the experimental distribution of Fig. 3(a) is remarkable. More specifically, a mono-exponential relaxation behavior is found both near the 'shoulders' and near the center of the Pake pattern, while a bi-exponential behavior is found near the 'horns'. Furthermore, the predicted coefficients $A_{i}$ shown in Fig 3(d) agree very well with the experimental ones of Fig. 3 (b). These simulations clearly indicate that our theoretical description of the observed relaxation rates of protons in water molecules trapped in crystals of barium chlorate monohydrate is very accurate.

Since the fluctuations of the CSA interactions provide the driving force of relaxation, we have repeated our relaxation measurements at $B_{0}=18.8 \mathrm{~T}\left(800 \mathrm{MHz}\right.$ for $\left.{ }^{1} \mathrm{H}\right)$ and, surprisingly, found substantially identical results, with a bi-exponential behavior of the integral over the entire width of the Pake pattern described by $T_{1}{ }^{(1)}=9.9 \pm 0.6 \mathrm{~s}$ and $T_{1}{ }^{(2)}=35.7 \pm 2.9 \mathrm{~s}$, bearing in mind that the orientation dependence is not affected by the static field. This can be rationalized by noting that the term $\left(\Delta_{C S} \omega_{0}\right)^{2}$ in Eq. (1) can be compensated by the term $3 k_{e x} /\left(4 k_{e x}^{2}+\omega_{0}^{2}\right)$ in Eq (2). Indeed, simulations show that for $k_{e x}=4.5 \times 10^{8} \mathrm{~s}^{-1}$ (i.e., 10 times slower than the value estimated at $\mathrm{T}=298 \mathrm{~K}$ by extrapolation in our earlier study), ${ }^{23}$ the relaxation rates are predicted to increase by no more than $10 \%$ between 400 and $800 \mathrm{MHz}$. This mismatch is reasonable if one considers that we did not observe changes in spectral features for temperatures above $200 \mathrm{~K}$.

Equations 1 and 2 and the plots of Fig. 2 indicate that a given crystallite orientation within the powdered sample is characterized by one and only one relaxation rate, and therefore by mono-exponential relaxation. This agrees with both the experimental and calculated data of Fig. 3, which clearly show that either only one or at most two $T_{1}$ values are required to interpret 
the relaxation behavior at any point in the Pake pattern. In order to get insight into this aspect, the numerical simulations of Fig. 3 (c-d) were carried out for some single crystallite orientations.

Figure $4\left(\right.$ a) shows some typical $T_{1}$ values calculated in the ranges $0^{\circ}<\alpha_{C R}<180^{\circ}$ and $0^{\circ}<$ $\beta_{C R}<90^{\circ}$, plotted along the horizontal axis according to their contributions to the Pake pattern. As previously stated, the $T_{1}$ values vary considerably at almost any position within the Pake pattern and seem to contradict our experimental evidence that it is sufficient to postulate a biexponential behavior to rationalize the decays of Fig. 3. Figure 4(b) shows six mono-exponential decays that reflect the $T_{1}$ values for the six equiprobable crystallites with $\beta_{C R}=90^{\circ}$ located near the low-frequency 'horn', as indicated by arrows in Fig. 4(a). A semi-logarithmic plot is shown in the inset. The red points in Figure 4(c) show the normalized sum of these six decays. An attempt to fit these red points to a mono-exponential decay is represented by a dashed blue line. A more satisfactory bi-exponential fit is shown by a red dashed line. A semi-logarithmic plot is shown in the inset. Clearly, despite the great range of time constants involved in the overall relaxation process at a given position within the Pake pattern, a simple bi-exponential fit suffices to describe the system. This finding fully supports our experimental profiles of Fig. 3 and the continuous distribution of $T_{1}$ values predicted in Fig. 2.

The mono-exponential relaxation behavior of individual crystallites was confirmed by means of ${ }^{1} \mathrm{H}$ spectra of a static single crystal. Figure 5 shows a series of dipolar doublets obtained for a small single crystal of $\mathrm{Ba}\left(\mathrm{ClO}_{3}\right)_{2} \cdot \mathrm{H}_{2} \mathrm{O}$ that was allowed to settle freely in a nonspinning $4 \mathrm{~mm}$ rotor as the latter was inserted into the probe. By repeatedly inserting and ejecting the rotor, we could obtain four 'arbitrary' orientations of the crystal with respect to the external field $B_{0}$. These doublets all have clearly distinct $T_{1}$ values, as expected by inspection of Fig. 2. Moreover, and in agreement with the theory outlined above, the recovery was always mono-exponential for each orientation of the single crystal.

\section{Conclusions}

The orientation dependence of the longitudinal relaxation time constants $T_{1}$ of protons in water molecules confined in crystals of barium chlorate monohydrate has been investigated by 
means of ${ }^{1} \mathrm{H}$ NMR spectroscopy in a static (non-spinning) polycrystalline powdered sample. By analyzing the role of modulation of the proton CSA interaction, this study offers new insights compared to the work by Long et al. ${ }^{29}$ on relaxation of deuterium in similar powders where relaxation is induced by modulations of much larger quadrupolar couplings. Some portions of the spectra near the 'horns' of the Pake pattern reveal a clear bi-exponential relaxation profile. In contrast, a simple mono-exponential recovery is found near the center and near the 'shoulders' of the Pake pattern. A theoretical description in Liouville space that takes into account the orientation dependence of $T_{1}$ and fast chemical exchange agrees very well with the experimental evidence. Each individual crystal orientation is predicted to have a purely monoexponential recovery behavior, which was confirmed by experiments on different orientations of a single crystal. The distribution of time constants and crystallite orientations found in a powder can be adequately interpreted with a simple bi-exponential model. We show that, in spite of fast hopping about the $\mathrm{H}-\mathrm{O}-\mathrm{H}$ bisector that requires the breaking of two hydrogen bonds, the magnetization of ortho water in crystallites of $\mathrm{Ba}\left(\mathrm{ClO}_{3}\right)_{2} \cdot \mathrm{H}_{2} \mathrm{O}$ at some orientations may have lifetimes comparable to, if not longer than, those of para-to-ortho conversion of water in fullerene cages. This study shows that the relaxation time constants of water molecules can be controlled by adjusting their orientation with respect to the external magnetic field.

\section{Acknowledgements}

The authors are indebted to Dr Mathieu Baudin and Dr Nicolas Birlirakis for assistance with experiments, and to Prof Nicolas Giraud for constructive comments. This work was supported by the CNRS, the European Research Council (ERC, contract 'dilute para-water', grant agreement number 339754), and the French "Equipements d'Excellence" Paris-en-Resonance, contract ANR-10-EQPX-09.

\section{References}

(1) Huang, X.; Xu, Y.; Karato, S. Water Content in the Transition Zone from Electrical Conductivity of Wadsleyite and Ringwoodite. Nature 2005, 434 (7034), 746-749.

(2) Schmandt, B.; Jacobsen, S. D.; Becker, T. W.; Liu, Z.; Dueker, K. G. Dehydration Melting at the Top of the Lower Mantle. Science. 2014, 344 (6189), 1265-1268.

(3) Griffin, J. M.; Berry, A. J.; Frost, D. J.; Wimperis, S.; Ashbrook, S. E. Water in the Earth's 
Mantle: A Solid-State NMR Study of Hydrous Wadsleyite. Chem. Sci. 2013, 4 (4), 15231538.

(4) Jacobsen, S. D. Effect of Water on the Equation of State of Nominally Anhydrous Minerals. Rev. Mineral. Geochemistry 2006, 62 (1), 321-342.

(5) Zhu, H.; Bozdağ, E.; Duffy, T. S.; Tromp, J. Seismic Attenuation beneath Europe and the North Atlantic: Implications for Water in the Mantle. Earth Planet. Sci. Lett. 2013, 381, 111.

(6) Harris, R. K. Applications of Solid-State NMR to Pharmaceutical Polymorphism and Related Matters. J. Pharm. Pharmacol. 2007, 59 (2), 225-239.

(7) Brittain, H. G. Polymorphism Solvatomorphism 2008. J. Pharm. Sci. 2010, 99 (9), 36483664.

(8) Halle, B. Protein Hydration Dynamics in Solution: A Critical Survey. Philos. Trans. R. Soc. London. Ser. B Biol. Sci. 2004, 359 (1448), 1207-1224.

(9) Gorshunov, B. P.; Zhukova, E. S.; Torgashev, V. I.; Lebedev, V. V; Shakurov, G. S.; Kremer, R. K.; Pestrjakov, E. V; Thomas, V. G.; Fursenko, D. A.; Dressel, M. Quantum Behavior of Water Molecules Confined to Nanocavities in Gemstones. J. Phys. Chem. Lett. 2013, 4 (12), 2015-2020.

(10) Kolesnikov, A. I.; Reiter, G. F.; Choudhury, N.; Prisk, T. R.; Mamontov, E.; Podlesnyak, A.; Ehlers, G.; Seel, A. G.; Wesolowski, D. J.; Anovitz, L. M. Quantum Tunneling of Water in Beryl: A New State of the Water Molecule. Phys. Rev. Lett. 2016, 116 (16), 167802.

(11) Richardson, J. O.; Pérez, C.; Lobsiger, S.; Reid, A. A.; Temelso, B.; Shields, G. C.; Kisiel, Z.; Wales, D. J.; Pate, B. H.; Althorpe, S. C. Concerted Hydrogen-Bond Breaking by Quantum Tunneling in the Water Hexamer Prism. Science. 2016, 351 (6279), 1310-1313.

(12) Torchia, D. A.; Szabo, A. Spin-Lattice Relaxation in Solids. J. Magn. Reson. 1982, 49 (1), 107-121.

(13) Mammoli, D.; Salvi, N.; Milani, J.; Buratto, R.; Bornet, A.; Sehgal, A. A.; Canet, E.; Pelupessy, P.; Carnevale, D.; Jannin, S.; Bodenhausen, G. Challenges of Preparing, Preserving and Detecting Para-Water in Bulk: Overcoming Proton Exchange and Other Hurdles. Phys. Chem. Chem. Phys. 2015, 17 (40), 26819-26827.

(14) Duer, M. J. Solid-State NMR Spectroscopy Principles and Applications; Blackwell Science Ltd: London, 2007.

(15) Carravetta, M.; Johannessen, O. G.; Levitt, M. H. Beyond the T1 Limit: Singlet Nuclear Spin States in Low Magnetic Fields. Phys. Rev. Lett. 2004, 92 (15), 153003.

(16) Eisendrath, H.; Stone, W.; Jeener, J. NMR of Protons in Gypsum. I. Experimental Proof of the Existence of Four Thermodynamic Invariants. Phys. Rev. B 1978, 17 (1), 47-53.

(17) Eisendrath, H.; Jeener, J. NMR of Protons in Gypsum. II. Independent Observation of Molecular Reorientations and Molecular Diffusion. Phys. Rev. B 1978, 17 (1), 54-60.

(18) Pake, G. E. Nuclear Resonance Absorption in Hydrated Crystals: Fine Structure of the Proton Line. J. Chem. Phys. 1948, 16 (4), 327-336.

(19) A Deuteron NMR Study on the Rotational Dynamics and the Orientation of Benzene Molecules Adsorbed on Graphite and Boron Nitride. Zeitschrift für Naturforschung $A$ 1986, $\mathrm{p} 1361$.

(20) A Deuteron NMR Study on a Benzene Multilayer on Graphite. Zeitschrift für Naturforschung A 1987, p 272. 
(21) Tekely, P.; Palmas, P.; Mutzenhardt, P. Influence of Proton Chemical-Shift Anisotropy on Magic-Angle Spinning Spectra of Hydrate Crystals. J. Magn. Reson. 1997, 127 (2), 238240.

(22) Carnevale, D.; Ashbrook, S. E.; Bodenhausen, G. Solid-State NMR Measurements and DFT Calculations of the Magnetic Shielding Tensors of Protons of Water Trapped in Barium Chlorate Monohydrate. RSC Adv. 2014, 4 (99), 56248-56258.

(23) Carnevale, D.; Pelupessy, P.; Bodenhausen, G. Cross-Term Splittings Due to the Orientational Inequivalence of Proton Magnetic Shielding Tensors: Do Water Molecules Trapped in Crystals Hop or Tunnel? J. Phys. Chem. Lett. 2019, 10 (12), 3224-3231.

(24) Bodenhausen, G.; Freeman, R.; Turner, D. L. Suppression of Artifacts in Two-Dimensional J Spectroscopy. J. Magn. Reson. 1977, 27 (3), 511-514.

(25) Antonijevic, S.; Wimperis, S. Refocussing of Chemical and Paramagnetic Shift Anisotropies in 2H NMR Using the Quadrupolar-Echo Experiment. J. Magn. Reson. 2003, 164 (2), 343350.

(26) Kartha, G. Structure of Barium Chlorate Monohydrate $\mathrm{Ba}(\mathrm{ClO} 3) 2 . \mathrm{H} 2 \mathrm{O}$. Acta Cryst. 1952, 5 (6), 845-846.

(27) Sikka, S. K.; Momin, S. N.; Rajagopal, H.; Chidambaram, R. Neutron-Diffraction Refinement of the Crystal Structure of Barium Chlorate Monohydrate $\mathrm{Ba}(\mathrm{ClO} 3) 2 \cdot \mathrm{H} 2 \mathrm{O}$. J. Chem. Phys. 1968, 48 (5), 1883-1889.

(28) Silvidi, A. A. Protonic Spin-Lattice Relaxation in Some Hydrated Barium Salt Crystals. J. Chem. Phys. 1966, 45 (10), 3892-3894.

(29) Long, J. R.; Ebelhäuser, R.; Griffin, R. G. 2H NMR Line Shapes and Spin-Lattice Relaxation in $\mathrm{Ba}(\mathrm{ClO} 3) 2 \cdot 2 \mathrm{H} 2 \mathrm{O}$. J. Phys. Chem. A 1997, 101 (6), 988-994.

(30) Keeler, E. G.; Michaelis, V. K.; Griffin, R. G. 170 NMR Investigation of Water Structure and Dynamics. J. Phys. Chem. B 2016, 120 (32), 7851-7858.

(31) Carnevale, D.; Chinthalapalli, S.; Bodenhausen, G. Exciting Wide NMR Spectra of Static Solid Samples with Weak Radiofrequency Fields. Z. Phys. Chem. 2017, 231, 527-543.

(32) Vold, R. R.; Vold, R. L. Deuterium Relaxation In Molecular Solids; WARREN, W. S., Ed.; Advances in Magnetic and Optical Resonance; Academic Press, 1991; Vol. 16, pp 85-171.

(33) Cavanagh, J.; Fairbrother, W. J.; Palmer, A. G.; Rance, M.; Skelton, N. J. Protein NMR Spectroscopy (Second Edition), Second Edi.; Academic Press: Burlington, 2007.

(34) Meier, B.; Kouřil, K.; Bengs, C.; Kouřilová, H.; Barker, T. C.; Elliott, S. J.; Alom, S.; Whitby, R. J.; Levitt, M. H. Spin-Isomer Conversion of Water at Room Temperature and QuantumRotor-Induced Nuclear Polarization in the Water-Endofullerene H2O@C60. Phys. Rev. Lett. 2018, 120 (26), 266001.

(35) Ernst, R. R.; Bodenhausen, G.; Wokaun, A. Principles of Nuclear Magnetic Resonance in One and Two Dimensions; Clarendon Press: Oxford, 1987.

(36) Duer, M. J.; Levitt, M. H. Time-Domain Calculation of Chemical Exchange Effects in the NMR Spectra of Rotating Solids. Solid State Nucl. Magn. Reson. 1992, 1 (4), 211-215. 
Figures

(a)

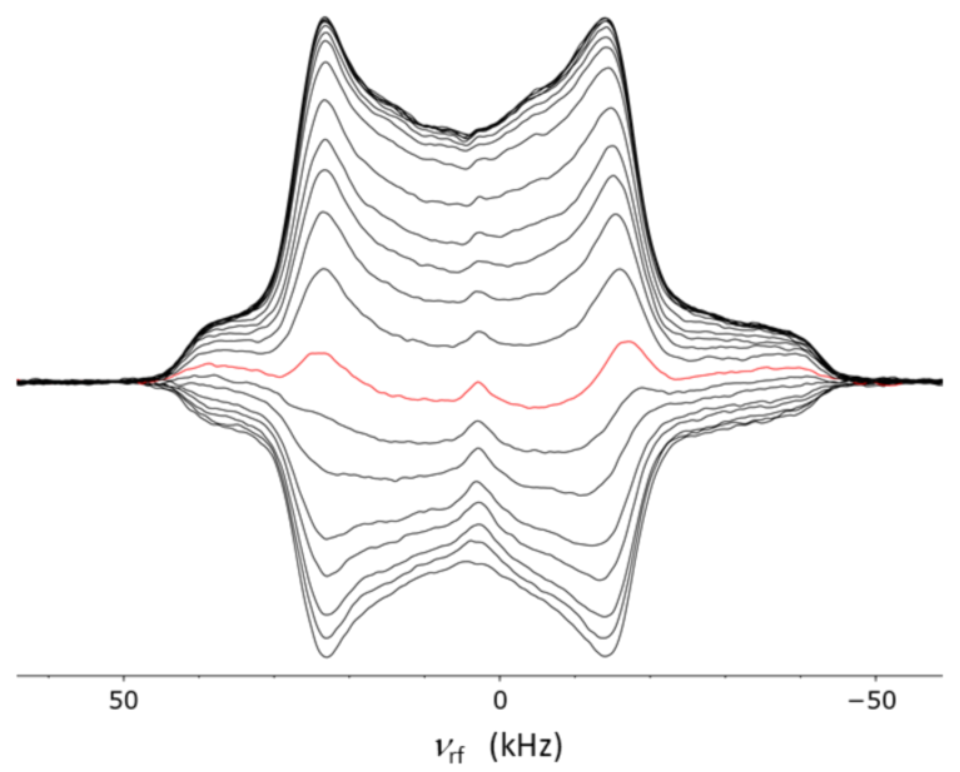

(b)

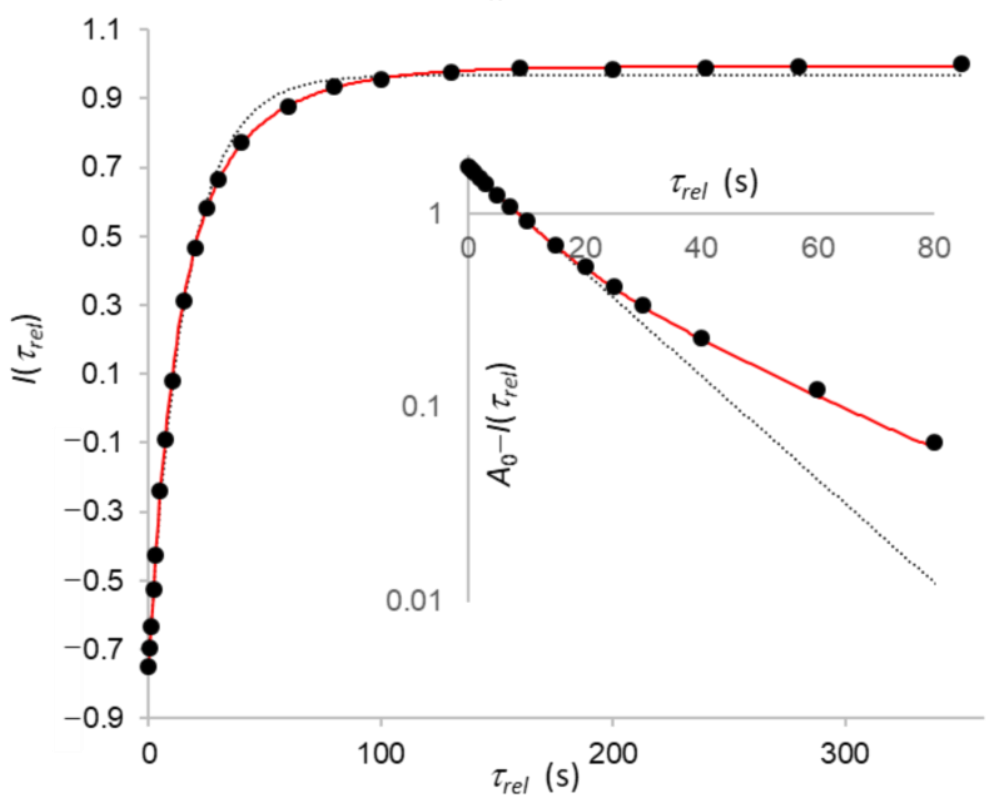

Figure 1 (a) Proton NMR spectra of a static powdered sample of $\mathrm{Ba}\left(\mathrm{ClO}_{3}\right)_{2} \cdot \mathrm{H}_{2} \mathrm{O}$ at $\mathrm{B}_{0}=9.4 \mathrm{~T}(400$ $\mathrm{MHz}$ for ${ }^{1} \mathrm{H}$ ) and $T=298 \mathrm{~K}$, observed with an inversion-recovery dipolar echo sequence $180^{\circ}-\tau_{\text {rel }}-90^{\circ}-\tau_{\mathrm{e}}$ - $90^{\circ}{ }_{\phi}-\tau_{\mathrm{e}}$ - acquisition, with $\tau_{\mathrm{e}}=30 \mu \mathrm{s}$. The $180^{\circ}$ and $90^{\circ}$ pulse widths were 5 and $2.5 \mu \mathrm{s}$, respectively. The phase of the last $90^{\circ}$ pulse was cycled through $\phi=\left\{0^{\circ}, 90^{\circ}, 180^{\circ}, 270^{\circ}\right\}$ while the receiver phase was alternated $\phi_{\mathrm{rec}}=\left\{0^{\circ}, 180^{\circ}, 0^{\circ}, 180^{\circ}\right\}$. Consecutive experiments were separated by a recovery interval of $30 \mathrm{~s}$. (b) Normalized signal intensity resulting from integration over the entire width of the Pake patterns in (a) as a function of the relaxation interval $\tau_{\text {rel. }}$. Experimental integrals are shown as black circles. An attempt to fit a mono-exponential function is represented by a dashed black line, whereas the continuous red line shows a bi-exponential fit to the function $I\left(\tau_{\text {rel }}\right)=A_{0}-A_{1} \exp \left\{-\tau_{\text {rel }} / T_{1}{ }^{(1)}\right\}-A_{2} \exp \{-$ $\tau_{\text {rel }} / T_{1}{ }^{(2)}$. An analogous semi-logarithmic plot is shown in the inset. 

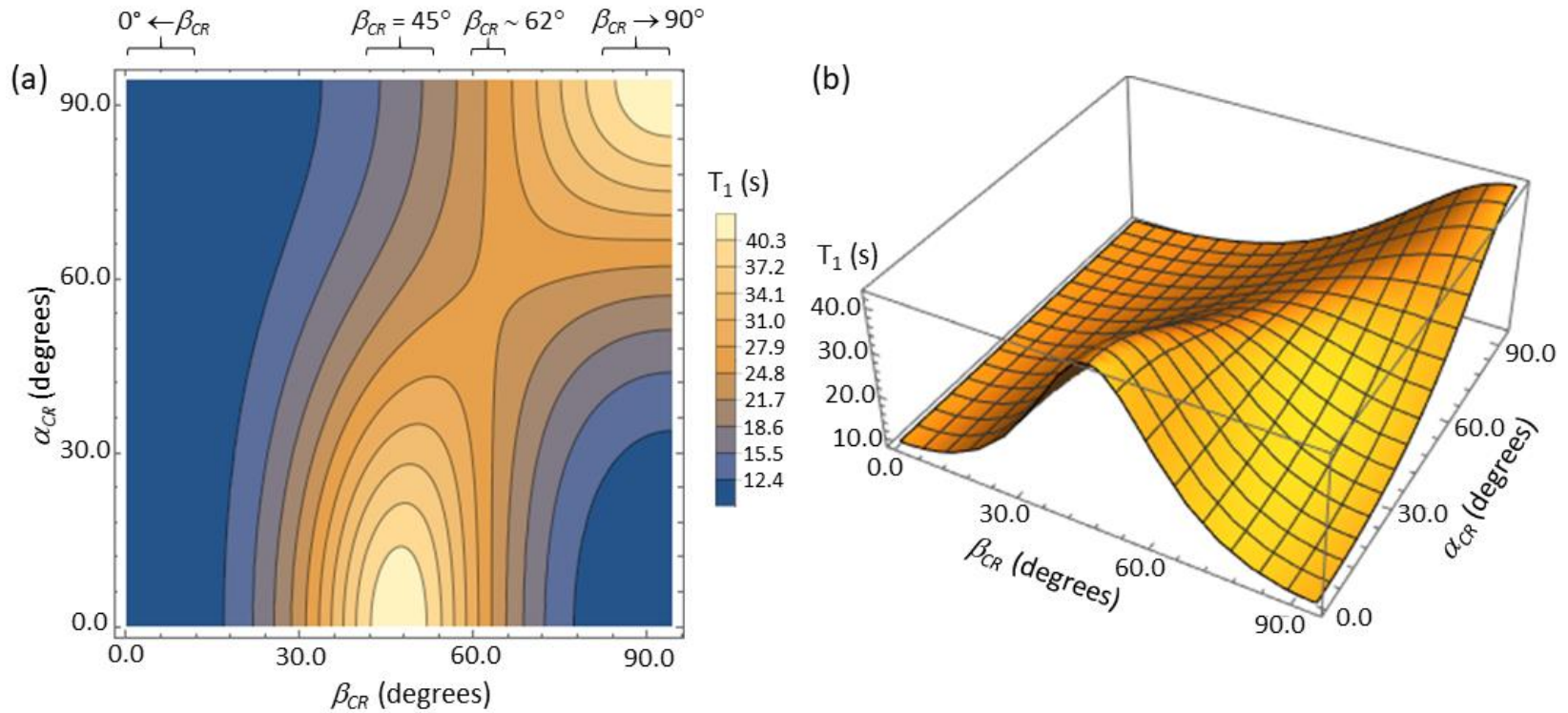

Figure 2 (a) Contour plot of the spectral density $J_{1}\left(\omega_{0}\right)$ of Eq. (2) that determines the longitudinal relaxation constant $T_{1}=1 / R_{1}$, obtained by Fourier transformation of the correlation function $C(\tau)$ of the fluctuations of the proton chemical shift anisotropy $\left({ }^{1} \mathrm{H} \mathrm{CSA}\right)$ at the Larmor frequency $\omega_{0} /(2 \pi)=400 \mathrm{MHz}$, as a function of the angles $\alpha_{C R}$ and $\beta_{C R}$ that describe the orientation of a crystallite and must be intergated for powder averaging. The exchange rate assumed at $298 \mathrm{~K}$ was $k_{\mathrm{ex}}=4.5 \times 10^{9} \mathrm{~Hz}$. (b) Threedimensional plot of the function shown in (a). 


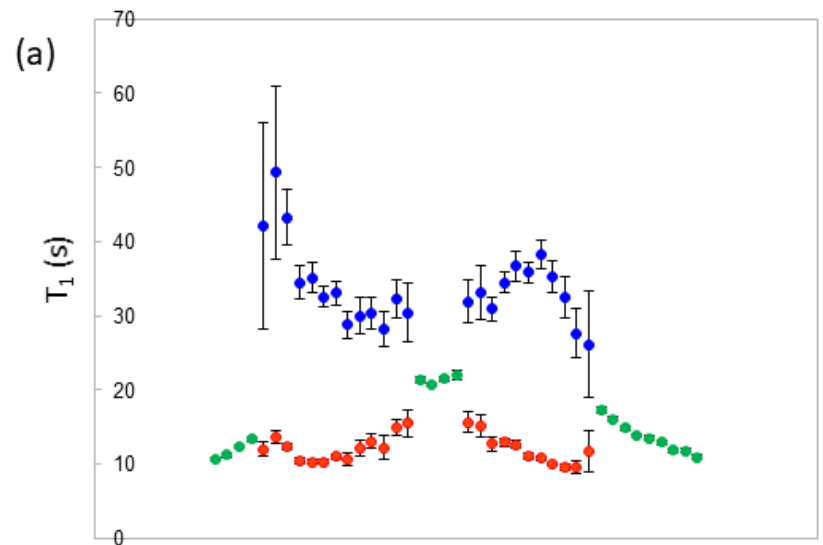

(c)
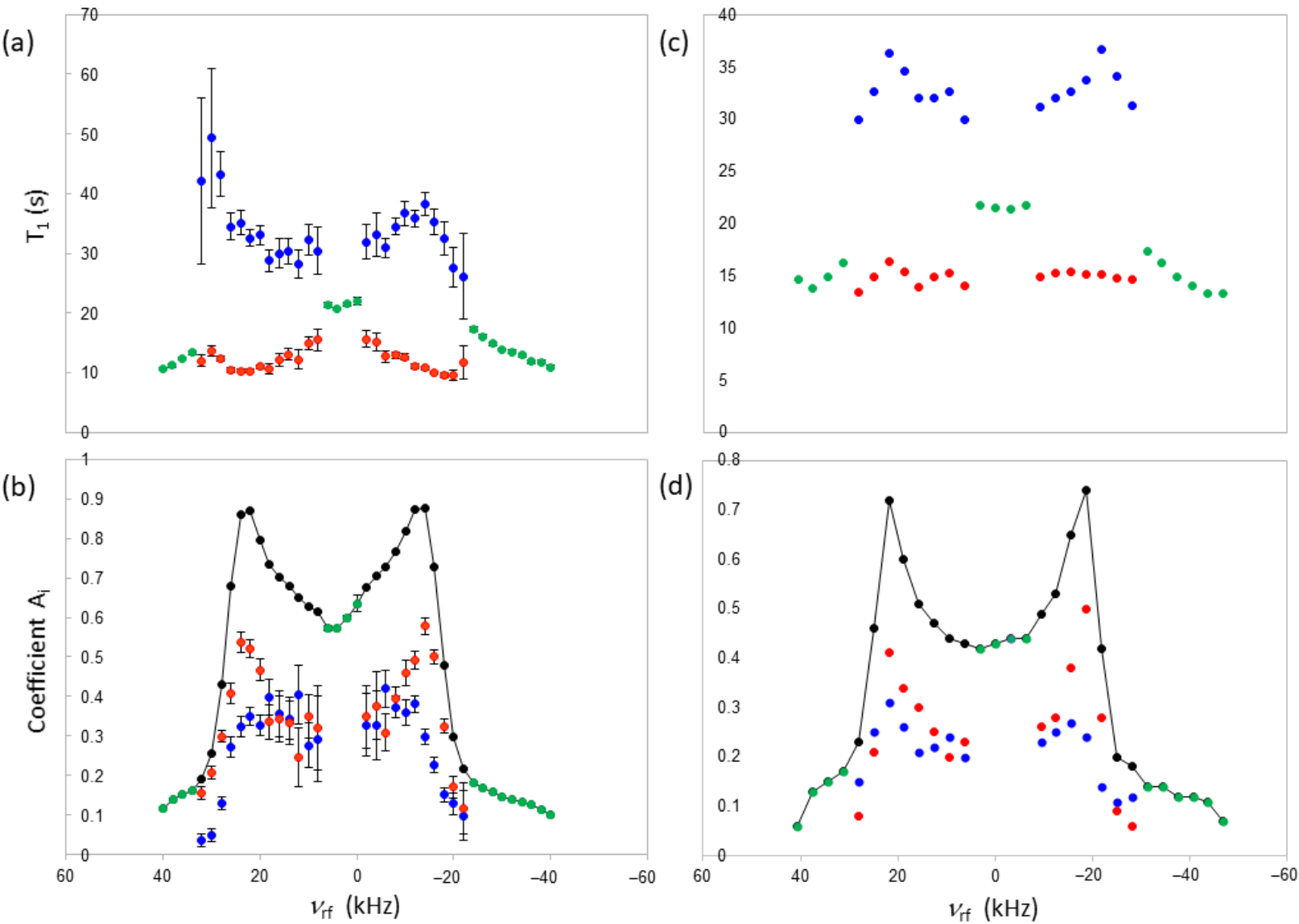

Figure 3 (a) Distribution of longitudinal relaxation time constants $T_{1}$ across the Pake pattern extracted from the inversion-recovery spectra of Fig. 1(a). When mono-exponential fitting was satisfactory, the resulting short relaxation time constants $T_{1}{ }^{(1)}$ are shown by green circles, whereas data resulting from bi-exponential fitting are represented by red and blue circles, for short and long relaxation time constants $T_{1}{ }^{(1)}=T_{1}$ (short) and $T_{1}{ }^{(2)}=T_{1}^{\text {(long) }}$, respectively. (b) Coefficients $A_{1}$ and $A_{2}$ of the fitting function $I\left(\tau_{\text {rel }}\right)=A_{0}-A_{1} \exp \left\{-\tau_{\text {rel }} / T_{1}{ }^{(1)}\right\}-A_{2} \exp \left\{-\tau_{\text {rel }} / T_{1}{ }^{(2)}\right\}$ shown using the same color code. The black circles indicate the sum $A_{1}+A_{2}$ of the weights. All experimental intensities are sampled in steps of $2 \mathrm{kHz}$ across the Pake pattern of Fig. 1a. (c) Calculated short and long relaxation time constants $T_{1}{ }^{(1)}=T_{1}{ }^{\text {(short) }}$ and $T_{1}{ }^{(2)}=T_{1}{ }^{\text {(long) }}$, with the same color codes as for the experimental values of (a), derived from the spectral density function of Eq. (2) shown in Fig. 2. (d) Calculated coefficients $A_{1}$ and $A_{2}$ with the same color codes. The data in (c, d) were calculated in steps of $3.125 \mathrm{kHz}$ across the Pake pattern of Fig. 1a. In (c, d), the rate of two-fold hopping about the $\mathrm{H}-\mathrm{O}-\mathrm{H}$ bisector was assumed to be $k_{e x}=4.5 \times 10^{9} \mathrm{~Hz}$ at $298 \mathrm{~K}$. Most error bars are smaller than the size of the data points. An isotropic contribution to the relaxation rates $R_{1}{ }^{\text {(iso) }}=$ $1 / T_{1}{ }^{\text {(iso) }}=0.018 \mathrm{~Hz}$ was included to take long-range intermolecular dipolar couplings and small librations into account. 
(a)

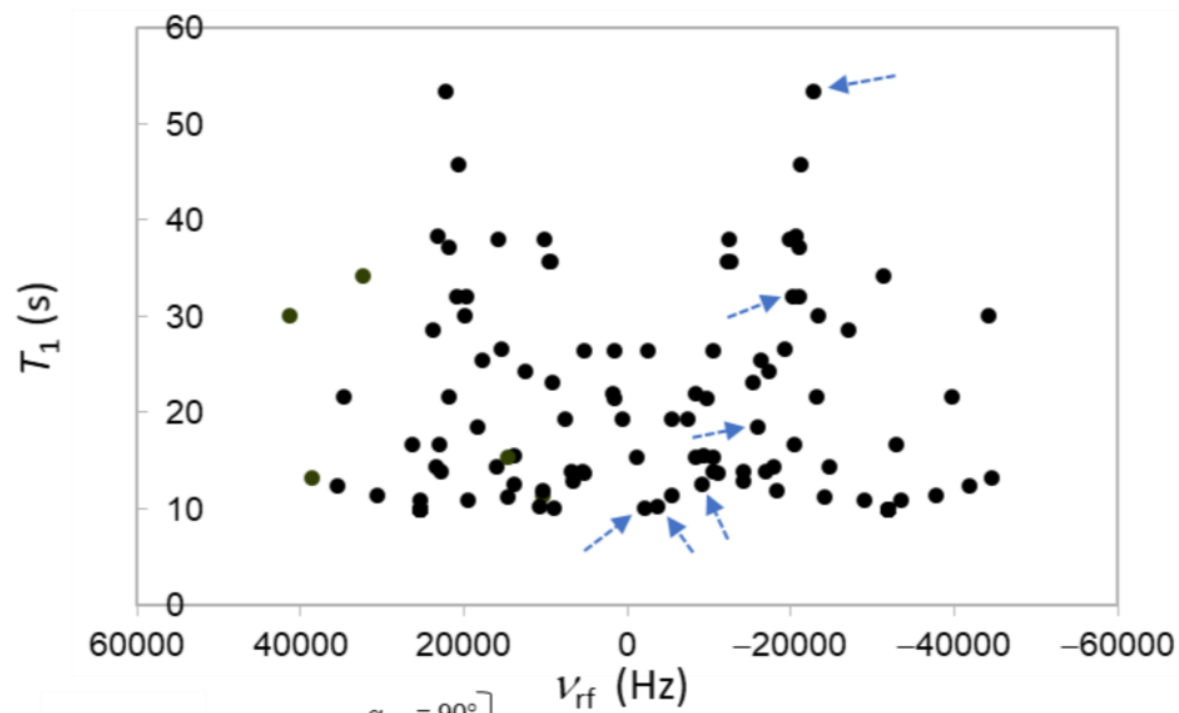

(b)

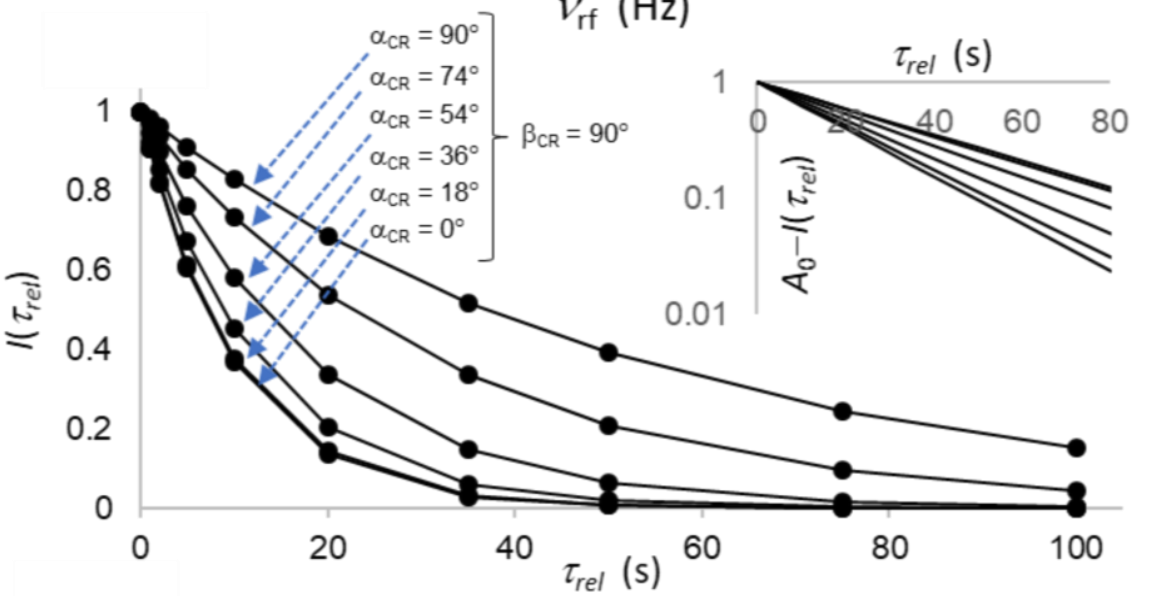

(c)

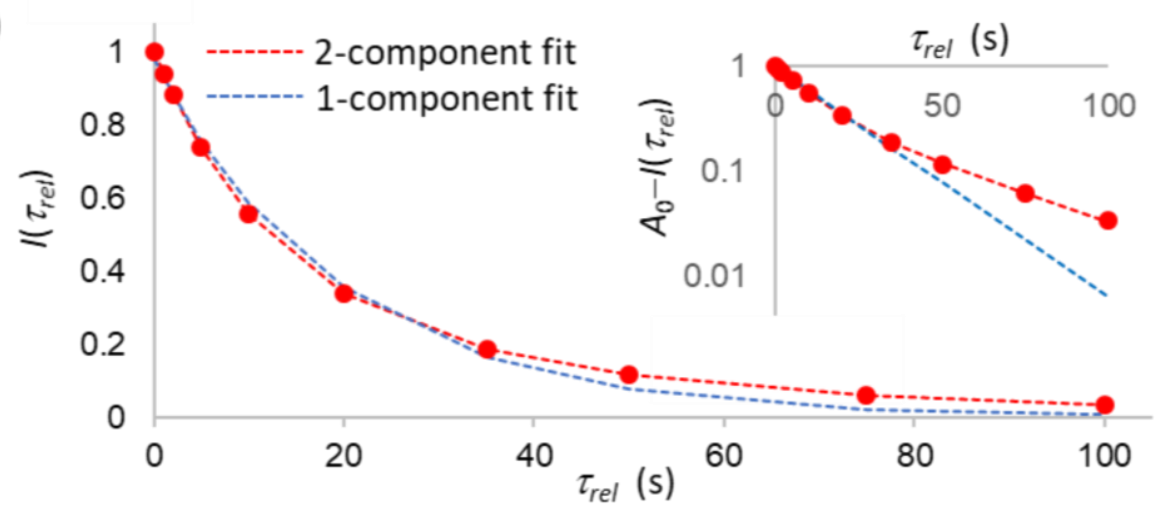

Figure 4 (a) Distribution of calculated relaxation time constants $T_{1}$-across the Pake pattern for 66 single crystallite orientations associated with $0^{\circ} \leq \beta_{\mathrm{CR}} \leq 90^{\circ}$ and $0^{\circ} \leq \alpha_{\mathrm{CR}} \leq 180^{\circ}$, in 5 and 10 steps of $18^{\circ}$, respectively. (b) Six simulated mono-exponential decays corresponding to the calculated $T_{1}$ values indicated by blue arrows in (a) for equiprobable crystallite orientations with $\beta_{\mathrm{CR}}=90^{\circ}$. An analogous semi-logarithmic plot is shown in the inset. (c) The red points indicate the normalized sum of the six decays of (b). A bi-exponential fit to these red points is shown by a red dashed line, whereas an attempt to fit a mono-exponential decay is represented by a blue dashed line. The inset shows a semi-logarithmic plot of the same data points. 


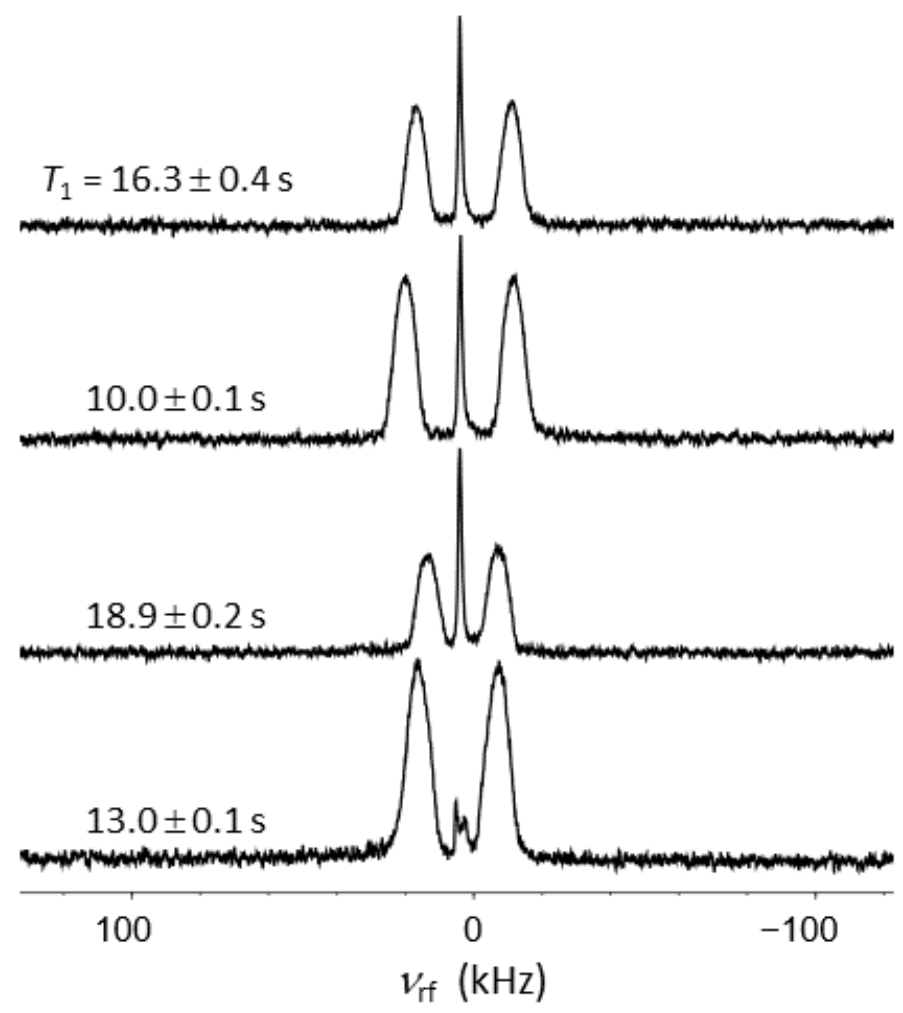

Figure 5 Experimental proton doublets of a single crystal of barium chlorate monohydrate $\mathrm{Ba}\left(\mathrm{ClO}_{3}\right)_{2} \cdot \mathrm{H}_{2} \mathrm{O}$ at $B_{0}=9.4 \mathrm{~T}\left(400 \mathrm{MHz}\right.$ for $\left.{ }^{1} \mathrm{H}\right)$ and $T=298 \mathrm{~K}$, observed at four different random orientations with respect to the external magnetic field. The sharp isotropic peaks appearing in the center of the doublets are attributed to water molecules that are loosely bound on the surface of the crystals. These peaks provide a measure of the homogeneity of the static field $\mathrm{B}_{0}$. Inversion-recovery experiments show monoexponential relaxation with time constants between 10 and $20 \mathrm{~s}$. 


\section{TOC Graphic}

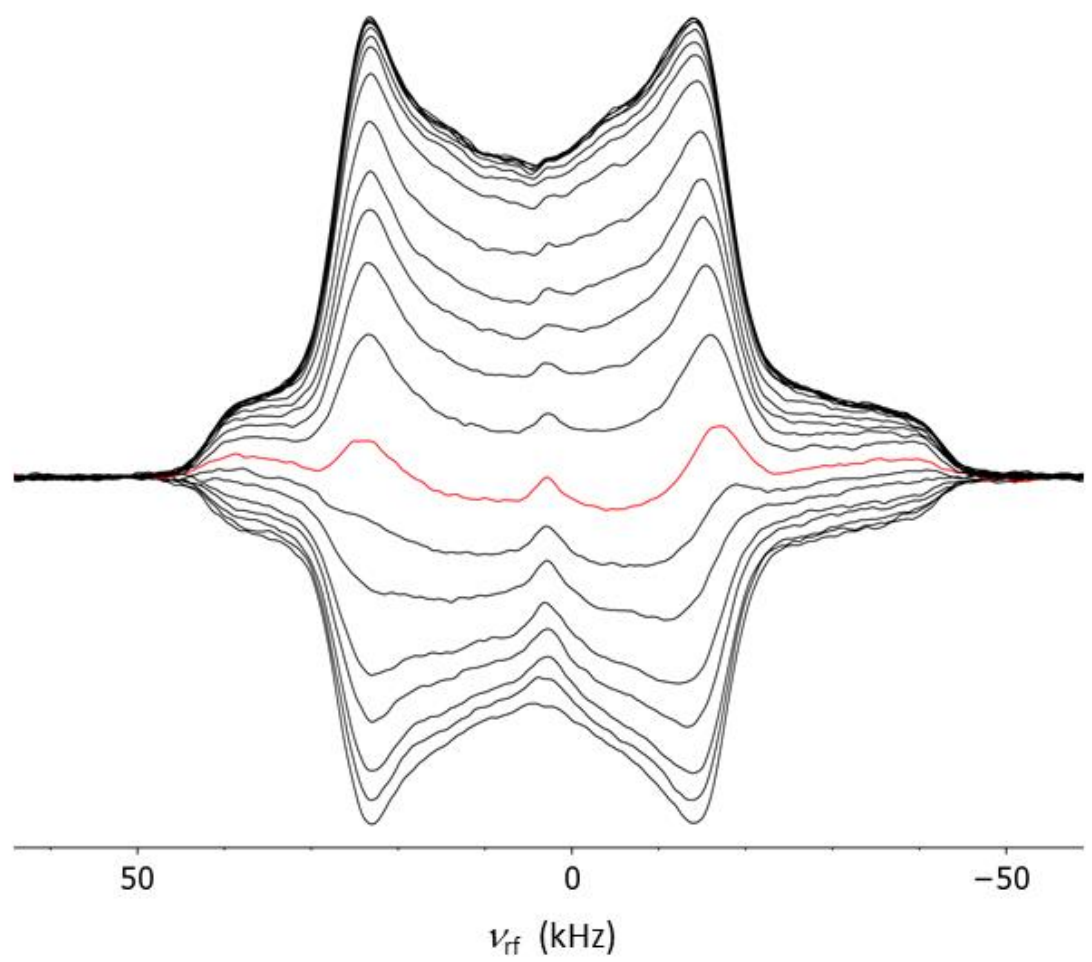

